\title{
Power Minimization under Real-Time Source Distortion Constraints in Wireless Networks
}

\author{
Andres Kwasinski, Zhu Han, K.J. Ray Liu and Nariman Farvardin \\ Department of Electrical and Computer Engineering, University of Maryland, College Park. \\ \{ankwasin,hanzhu,kjrliu,farvar\}@eng.umd.edu
}

\begin{abstract}
In multiple access wireless networks with co-channel interference, allocating resources such as transmitted powers and source rates is a task critical to improve performance. In this paper, we introduce a new technique aimed at minimizing the overall transmitted power subject to constraints on the incurred source distortion. The technique is based on the use of real-time source codecs with externally adaptable output rate and Rate Compatible Punctured Convolutional (RCPC) channel encoders. We develop an adaptive algorithm to find the optimal power and source rate allocation to the different users according to their channel conditions and under distortion constraints. We present simulation results to show that it is possible to reduce the overall transmitted power significantly with a relatively small distortion increases for some users, and while keeping the network average distortion unchanged.
\end{abstract}

\section{INTRODUCTION}

In wireless networks, allocating resources such as transmitted powers and source rates is an important mean to combat cochannel interference, increase overall throughput, and maintain the transmitted signal quality. In power-controlled networks [1] [2], the transmitted powers are constantly adjusted. This process maintains each link's Signal-to-Interference plus noise ratio (SINR) within acceptable levels but, at the same time, increases the cochannel interferences during deep fade periods. It has been shown that system performance can be greatly improved by alleviating the fixed targeted SINR for each user [5]. A related idea, used to reduce interference in voice communications, is to take advantage of changes in speech activity levels by using variable-rate source encoders. This is the case of the IS-95 standard. In IS-95 the vocoder used, named QCELP, have four different operating states, each of them associated with a different level of speech energy and a different output rate. The vocoder is used in conjunction to the power allocation algorithm to reduce the transmit power when the source rate decreases. Nevertheless, this technique suffers from the limitation that the source rate changes are driven by the source, i.e. there is no external control over it. In this paper we introduce a technique based on using externally controllable variable rate source encoders. This allows us to control the targeted transmitted power by changing the source rate while also being able to control the incurred distortion.

In this paper, we study how to allocate transmitted power and source rate to the users in a wireless network. Power is allocated based on maintaining each user SINR above a thresh- old. In the case of users operating in deep fading channel conditions, the straightforward approach to assigning power would significantly increase the transmit power. Our approach considers that if these users could accept a controlled small degradation in source quality, the corresponding reduced rate and power assignment could greatly decrease cochannel interference and improve the overall network performance. Our algorithm minimizes the overall transmitted powers at the cost of a smooth controllable degradation of reconstructed source quality for the user with bad channel condition, while keeping the average network distortion unchanged. This solution is an across-layer design where, for each user, the rate is decided based on its channel condition while the distortion is bounded by a maximum allowable value. From simulation results, our algorithm reduces the total transmitted power of mobile users up to $80 \%$ relative to the scheme when all users have the minimum distortion. Furthermore, the overall power can be reduced up to $13 \%$ relative to the scheme where all the users have the same source rate and the same distortion, equal to the target average distortion [1].

The organization of this paper is as follows: In Section II, we give a description about our cross-layer protocol. In Section III, we present system model and the optimization problem is formulated. In section IV, we develop an adaptive algorithm for power minimization by controlling real-time source rate of different users. In Section V, we evaluate the performance of our algorithms through a simulation study. Section VI presents the conclusion.

\section{System DesCRIPTION}

Figure 1 shows a block diagram of the scheme that allows our proposed protocol to manage the interference by controlling the users' source rates and transmitted powers. The protocol is located in a centralized position (namely the base station) and communicates with all the mobiles in the coverage area. The protocol allocates rate and power to all the users based on the channel conditions in such a way that the SINR remains above a threshold. This threshold is determined by considering that the source Frame-Error-Rate (FER) is such that the distortion due to channel-induced errors should be within a range of acceptable small values. In doing so, the protocol also considers the effects on the reconstructed source quality. 
In our scheme, the real time source encoder has the key property that the output rate can be externally controlled regardless of the speech activity level. This can be implemented using either variable rate or embedded encoders. In the first case, the coder generates one bit stream for each of the possible encoding rates. Only one of these will be selected and transmitted based on the rate assignment. Using embedded encoders presents the advantage that only one bit stream is generated, making the adaptation to the rate assignment simple by dropping as many bits as necessary from the end of the bit stream. Although the "bit dropping mechanism" is exclusive to the embedded stream, we will loosely use this term to represent a reduction in the source rate regardless of the particular source encoder implementation.

In the proposed system, the channel error protection consists of two concatenated channel coders: one that is fixed and another that is variable rate. The fixed channel encoder with rate $1 / M$ operates with a constant input bit rate $R$ equal to the source encoder maximum output rate; all bits dropped from the source are replaced by error protection bits generated by the variable-rate channel encoder. Therefore, the source encoder output rate reduction $l$ is matched by an increased error protection of the transmitted source data.

For simplicity, we assume that all the source bits are equally important for error protection purposes. Our design is constrained by the condition to guarantee that a maximum source FER is not exceeded. There is a direct relationship between FER and SINR requirements because the power assigned to each mobile should be such that the resulting SINR guarantees a maximum source FER. Note that the SINR required to maintain a maximum source FER is a function of the source rate because reducing this rate corresponds to increasing error protection for the source frame which, in turn, reduces the required SINR. Therefore, it is possible to reduce the overall transmitted power in the network by reducing some or all users' source rate, an action that reduces the transmitted power and the interference but increase the source distortion. We will study how to allocate each user's rate to have minimum overall transmitted power, while keeping the network average source distortion performance unchanged and subject to a limit on the maximum distortion per user.

\section{System Model ANd Problem Formulation}

Consider K co-channel links that may exist in distinct cells. Each link consists of a mobile and its assigned base station. Assume coherent detection is possible so that it is sufficient to model this multiuser system by an equivalent baseband model. We assume that each link is affected by an additive, white Gaussian noise (AWGN) channel and that a matched filter is used in the receiver. The sampled received signal can be given by:

$$
x_{i}(n)=\sum_{k=1}^{K} \sqrt{P_{k} G_{k i}} s_{k}(n)+n_{i}(n),
$$

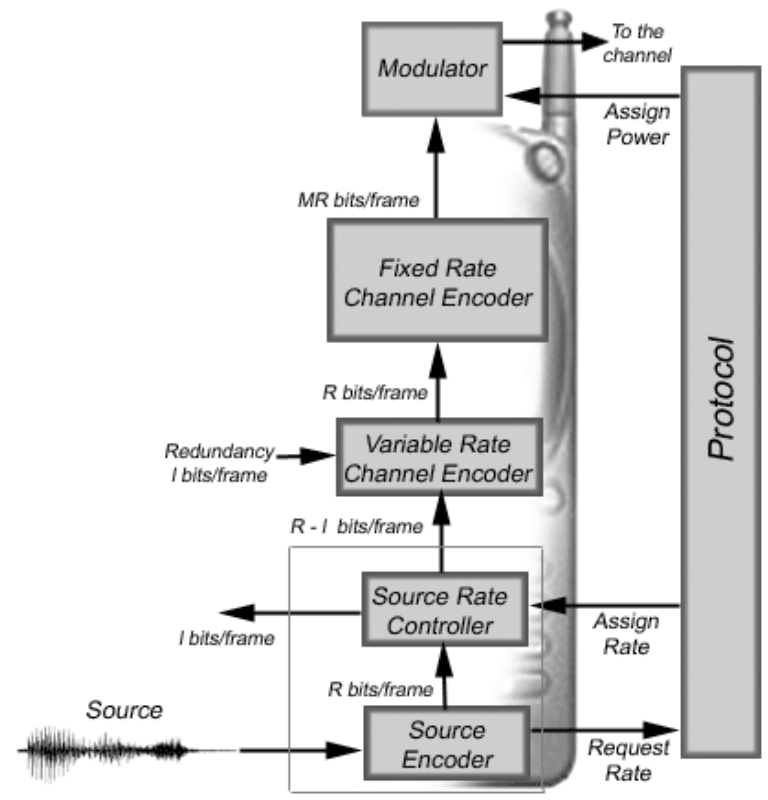

Fig. 1. Block diagram of the proposed system

where $P_{k}$ is the $k^{t h}$ link's transmitted power, $G_{k i}$ is the path loss for $k^{t h}$ user to $i^{\text {th }}$ base station, $s_{k}(n s)$ is the message symbol, and $n_{i}(n)$ is the sampled thermal noise. The $i^{t h}$ base station's output SINR is:

$$
\Gamma_{i}=\frac{P_{i} G_{i i}}{\sum_{k \neq i} P_{k} G_{k i}+N_{i}},
$$

where $N_{i}=E\left(\left\|n_{i}\right\|^{2}\right)$. Write equation (2) as a function of $P_{i}, i=1, \ldots, K$ in the matrix form:

$$
(\mathbf{I}-\mathbf{D F}) \mathbf{p}=\mathbf{u},
$$

where $\mathbf{p}=\left[P_{1}, \ldots, P_{K}\right]^{T}, \mathbf{u}=\left[u_{1}, \ldots, u_{K}\right]^{T}, u_{i}=$ $\Gamma_{i} N_{i} / G_{i i}, \mathbf{D}=\operatorname{diag}\left\{\Gamma_{1}, \ldots, \Gamma_{K}\right\}$, and

$$
\left[F_{i j}\right]= \begin{cases}0 & \text { if } j=i, \\ \frac{G_{j i}}{G_{i i}} & \text { if } j \neq i .\end{cases}
$$

The transmitted power vector in (3) depends on the targeted SINR, which is a function of the source rate. Following the system model presented in section II, it is clear that the target SINR will increase as the source rate grows (because the channel error protection decreases). Furthermore, we have found through simulations using different configurations of concatenated convolutional and Rate Compatible Punctured Convolutional (RCPC) codes that the SINR as a function of source rate can be approximated very accurately by the function

$$
\Gamma(r)=2^{A r+B},
$$

where $A$ and $B$ are the parameters of the error control coding scheme and $r$ is the source rate.

Define $f_{i}\left(r_{i}\right)$ as the distortion-rate function of the $i^{t h}$ source encoder transmitting at rate $r_{i}$. In most well designed encoders 
$f_{i}\left(r_{i}\right)$ is a convex and decreasing function. Even more, in good coders the minimum distortion occurs at maximum source rate. Also, for simplicity, we will also assume that the rates $r_{i}, \forall i$ are real numbers. Furthermore, we assume that the source encoder distortion-rate function is [9], [10]:

$$
f_{i}\left(r_{i}\right)=\delta 2^{2 k\left(r_{\max }-r_{i}\right)},
$$

where $\delta$ is the minimum distortion, $r_{\max }$ is the maximum achievable rate. This is a very general form that applies for the case of Gaussian source with squared-error distortion or when the high-rate approximation holds. Furthermore, in the case of realistic encoders we found that (6) constitute a good and tight upper bound on the real distortion-rate curve. The parameter $k$ can be determined through simulations for any encoder so that (6) is a tight bound on the real distortion-rate operating curve. Define the normalized distortion $D_{i}=f_{i}\left(r_{i}\right) / \delta$. In our proposed system, we want to minimize the overall transmitted power under the constraints that each user's distortion is within an acceptable range and network average distortion is unchanged. The optimization problem formulation can be given by:

$$
\begin{gathered}
\qquad \min _{\Gamma_{i}} \sum_{i=1}^{K} P_{i} \\
\text { subject to }\left\{\begin{array}{l}
\text { Feasibility: }(\mathbf{I}-\mathbf{D F}) \mathbf{p} \geq \mathbf{u}, \\
\text { Distortion Range: } 1 \leq D_{i} \leq D_{\max }, \forall i, \\
\text { Average Distortion: } \frac{1}{K} \sum_{i=1}^{K} D_{i}=\Delta,
\end{array}\right.
\end{gathered}
$$

where $\Delta$ is the average network distortion and $D_{\max }$ is the maximum distortion that each user can accept.

\section{Power Minimization by Real-Time SOURCE RATE AdAPTATION}

In this section, we will use the projected gradient method [4] to have an algorithmic solution for (7). An adaptive algorithm will be developed to find each user's source rate so as to generate the lowest overall transmitted power under the constraints.

From (5) and (6), the distortion as a function of each user's targeted SINR is given by:

$$
D_{i}=2^{2 k\left(r_{\max }+B / A\right)}\left(\Gamma_{i}\right)^{-2 k / A} .
$$

When the user experiences bad channel conditions, it is assigned an operating point corresponding to a smaller source rate and larger source distortion. From (8), the user in this situation will select a smaller targeted SINR and, consequently, the overall transmitted power will be reduced. In the absence of any constraint, there is a clear possibility that users in very bad channel conditions would be commanded to reduce the source rate to the point where the corresponding source distortion would become excessive. Because of this reason, it is clear that rate and power adaptation should be constrained so that the distortion for all users remains within acceptable levels during the whole duration of the call. This constrain is included in our formulation by maintaining the average distortion at a constant level and by setting a maximum and minimum limit on each user's distortion (see (7)).

We assume that each link can accept a normalized source distortion in the range $\left[1, D_{\max }\right]$. Then each user's targeted SINR would be within the range

$$
\Gamma_{\min }=D_{\max }^{-\frac{A}{2 k}} 2^{A r_{\max }+B} \leq \Gamma_{i} \leq 2^{A r_{\max }+B}=\Gamma_{\max }
$$

Our problem becomes how to find each user's targeted SINR to generate the lowest overall constrained power. In [5], the author proves that $P_{\text {sum }}=\sum_{i=1}^{K} P_{i}$ is a convex and increasing function of $\Gamma_{i}$, when $\Gamma_{j}, j=1 \ldots K, j \neq i$ is fixed. We can use the projected gradient method [4] to adjust each link's targeted SINR threshold to reduce the overall transmitted power. $P_{\text {sum }}$ can be written as:

$$
P_{\text {sum }}=\mathbf{1}^{T}(\mathbf{I}-\mathbf{D F})^{-1} \mathbf{u}
$$

where $\mathbf{1}=[1 \ldots 1]^{T}$. Take derivatives with respect to SINR $\Gamma_{i}$ at both sides, then we have the $i^{\text {th }}$ element of gradient vector $\mathbf{g}=\left[g_{1} \ldots g_{K}\right]^{T}$ of $P_{\text {sum }}$ as[3]:

$$
g_{i}=\frac{\partial P_{s u m}}{\partial \Gamma_{i}}=\frac{C_{i} P_{i}}{\Gamma_{i}}
$$

where $C_{i}=\mathbf{1}^{T}(\mathbf{I}-\mathbf{D F})^{-1} \hat{\mathbf{v}}_{i}$, and $\left[\hat{\mathbf{v}}_{i}\right]_{j}=1$, if $j=i ; 0$ otherwise. The value of $C_{i}$ reflects how severe the co-channel interference is. When the co-channel interferences are large, $C_{i}$ tells which user causes more co-channel interferences to other users. When the co-channel interferences are small, $C_{i} \approx C_{j} \forall i, j$.

We also have a constraint on the average distortion condition $(1 / K) \sum_{i=1}^{K} D_{i}=\Delta$. If we change the targeted SINR by the gradient of (10), this constraint will not necessarily be satisfied. We need to modify the gradient. This is achieved by projecting the gradient onto the plane that is tangent to the curve $1 / K \sum_{i=1}^{K} D_{i}=\Delta$ at point $\left[\Gamma_{1}, \ldots, \Gamma_{K}\right]$. The plane can be expressed as: $\sum_{i=1}^{K} k_{i} x_{i}=$ const., where $k_{i}=-\left(\Gamma_{i}\right)^{-\frac{2 k}{A}-1}$. The modified gradient is given by $\mathbf{q}=\left[q_{1} \ldots q_{K}\right]^{T}$. By the definition of projection, vector $\mathbf{q}$ satisfies equation $\|\mathbf{g}-\mathbf{q}\|^{2}=$ $\min _{\forall \mathbf{x} \in \text { plane }}\|\mathbf{g}-\mathbf{x}\|^{2}$. We only need to minimize the right hand side to get the optimal vector,i.e. the projection $\mathbf{q}$ [5].

We can construct the adaptive iterative algorithm to find the best power and source rate allocation in Table I, where $\mu$ is a small constant, whose value decides the rate of convergence and the variance of final result. The algorithm is initialized by setting all users rate and SINR to the same operating point corresponding to the normalized distortion equal to the target average distortion. Then, the algorithm try to control each user's rate and distortion such that the overall power is decreased. The iteration is stopped when $\Gamma_{i}$ is stable for all $i$ or when any of the $\Gamma_{i}$ reaches a boundary corresponding to the per-user distortion constraint. Whether or not $\Gamma_{i}$ is stable is decided by comparing the maximum difference of $\Gamma_{i}$ in two consecutive steps. Following the similar proof in [2], it can be shown the algorithm is convergent. 
TABLE I

Adaptive Algorithm for SINR Allocation

\begin{tabular}{|l|}
\hline Initial: \\
\hline$\Gamma_{1}, \ldots \Gamma_{K}=\Delta^{-\frac{A}{2 k}} 2^{A r_{m a x}+B}$ \\
\hline Iteration: \\
Power Allocation Update Iteration: \\
p $=$ DFp $+\mathbf{u}$. \\
\hline Adaptive Allocation \\
do \{ \\
$\quad \mathbf{g}=\nabla P_{\text {sum }} ;$ \\
q $=$ projection $(\mathbf{g}) ;$ \\
$\Gamma_{i}=\Gamma_{i}-\mu . \mathbf{q}_{i} \forall i ;$ \\
if $\left(\Gamma_{i}>\Gamma_{\max }\right) \quad \Gamma_{i}=\Gamma_{\max }$, break; \\
if $\left(\Gamma_{i}<\Gamma_{\min }\right) \quad \Gamma_{i}=\Gamma_{\min }$, break. $\}$ \\
while $\left(\Gamma_{i}\right.$ not stable $)$ \\
\hline
\end{tabular}

\section{Simulation Results}

In order to evaluate the performance of our algorithm, we simulated a network with 50 hexagonal cells, each with a $1000 \mathrm{~m}$ radius. Two adjacent cells do not share the same channel. In each cell, one base station was placed at the center and one user was placed randomly with a uniform distribution. To obtain the average received power as a function of distance we used the two slopes path loss model [8]. We assumed the mobile antenna height was $2 \mathrm{~m}$ and the base station antenna height was $50 \mathrm{~m}$. The carrier frequency was $900-\mathrm{MHz}$.

The source encoder used was a variable-rate, single-state coder, derived from the QCELP encoder. It generates an

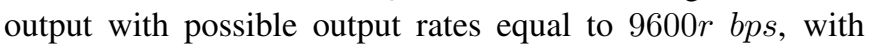
$r=\{1,7 / 8,6 / 8,5 / 8,4 / 8,3 / 8,2 / 8\}$. The source information in each $20 \mathrm{~ms}$ period is assumed to be packed into 2 to 8 packets, depending on the rate. Measurement of the mean squared-error rate-distortion function yielded $k=0.1$ (see (6)), when the rate is measured in terms of source packets per frame. Following the scheme in Figure 1, we concatenated a variable-rate and a fixed-rate channel encoder. The fixedrate channel encoder was implemented with the memory-two, rate $1 / 2$, convolutional encoder with the highest free distance [7]. A memory-four, puncturing period eight, RCPC code [6] was used for the variable-rate channel encoder block. In order to limit the distortion introduced by channel-induced errors to negligible values we set the maximum FER thresholds to $10^{-2}$ when the output rate was $r_{\max }$ and $10^{-3}$ otherwise. The maximum and minimum normalized distortion per user was set to 1.3 and 1 , respectively.

Figure 2 shows the average total power obtained using our algorithm relative to the total power with minimum distortion, i.e considering that all users transmit at maximum rate $r_{\max }$. We can see that the reduction is significant, increasing with the acceptable normalized average distortion up to approximately $80 \%$ reduction for a $25 \%$ increase in average normalized distortion.

Figure 3 shows the average total power obtained using our

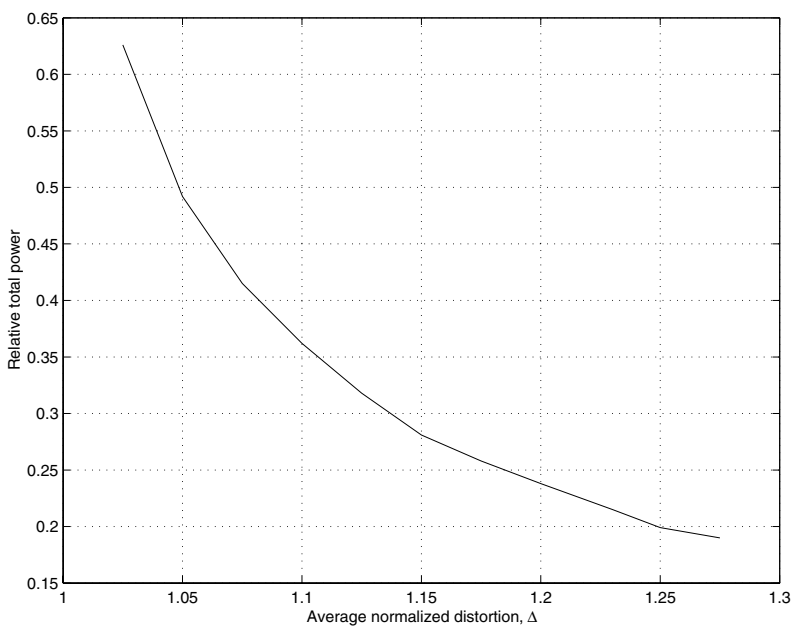

Fig. 2. Total power obtained using the algorithm relative to the total power without any adaptation as a function of normalized average distortion

algorithm relative to the total power when each user has the same rate $r=r_{\max }-\log _{2} \Delta /(2 k)$, i.e all users have the same distortion equal to $\Delta$. Here, we can see that our algorithm achieves gain values ranging from $13 \%$ for small normalized average distortion to $5 \%$ for large normalized average distortion values. Our algorithm reduces cochannel interference by reducing the users source rate. This reduction is more significant for users in worse channel conditions but is also subject to the distortion constraints discussed above. As the target average distortion is increased, the maximum distortion per user constraint limits the source rate reduction achievable for users in deep fade conditions. Therefore, the improved achievable with our algorithm is reduced as the target average reduction approaches the maximum distortion per user because the algorithm is more limited in the range of rates it can choose when adapting power and SINR.

The improvement provided by our algorithm depends on the channel conditions. In Figure 4 we show the histogram of the total power obtained using our algorithm relative to the total power with minimum distortion where $\Delta=1.1$. In most of the cases, the overall powers are reduced by $50 \%$ to $70 \%$.

\section{CONClusions}

In this paper we have proposed a novel adaptive algorithm with variable-rate source coding and RCPC channel coding for power minimization under distortion constraints in wireless networks. By proposing an across-layers design that adaptively manages link quality and transmitted power through source rate adaptation, we are able to improve network performance and reduce power consumption while considering the effects on source distortion at the same time.

From our simulation results, the algorithm reduces the total transmitted power of mobile users up to $80 \%$ relative to the total power with minimum distortion and up to $13 \%$ relative to the scheme where all the users have the same source rate and the same distortion, equal to the target average distortion $\Delta$. 


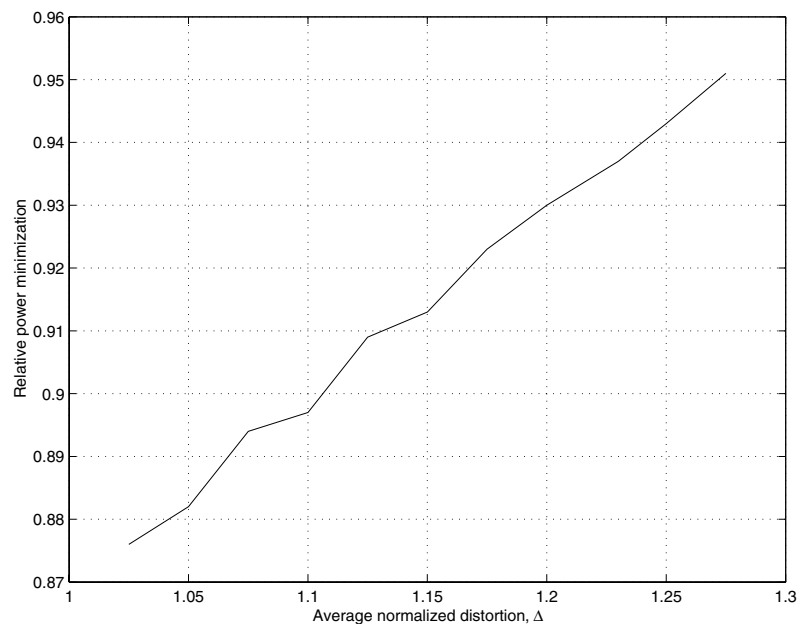

Fig. 3. Total power obtained using the algorithm relative to the total power allocated when all users operate at rate $r=r_{\max }-\log _{2} \Delta /(2 k)$

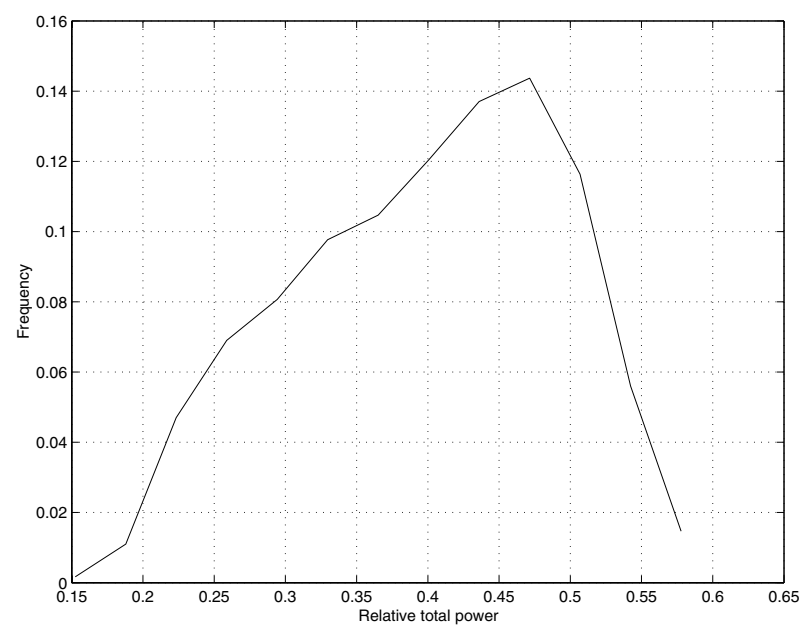

Fig. 4. Histogram of the total power obtained using our algorithm relative to the total power without any adaptation for the case of $\Delta=1.15$

These results are significant in terms of battery life in mobile sets and overall capacity for the whole networks.

\section{REFERENCES}

[1] F.Rashid-Farrokhi, L. Tassiulas, K.J. R. Liu, "Joint optimal power control and beamforming in wireless network using antenna arrays", IEEE Trans. Commun., vol.46, no.10, pp.1313-1323, Oct. 1998 .

[2] R. Yates, "A framework for uplink power control in cellular radio systems", IEEE J. Sel. Areas Commun., vol.13, no.7, pp.1341-1348, Sep. 1995.

[3] Z. Han, K.J.Ray Liu, "Adaptive coding for joint power control and beamforming over wireless networks", SPIE02, Seattle, July 2001.

[4] S. Boyd and L. Vandenberghe, "Introduction to convex optimization with engineering applications", [Online], http://www.stanford.edu/class/ee364/, 1999.

[5] Z. Han, K.J.R. Liu, "Adaptive SINR threshold allocation for joint power control and beamforming over wireless network", IEEE VTC., pp.15481552, Fall 2001.

[6] J. Hagenauer, "Rate compatible punctured convolutional (RCPC) codes and their applications", IEEE Transactions on Communications, vol. 36, pp.389-399, April 1988.

[7] J. G. Proakis, Digital Communications, McGraw-Hill Inc., 1994
[8] P. Harley, "Short distance attenuation measurements at 900MHz and 1.8 GHz using low antenna heights for microcells", IEEE Trans. Sel. Areas Commun., vol.37, pp.220-222, Nov. 1988.

[9] A Gersho, R. M. Gray, Vector Quantization and Signal Compression, Kluwer Academic Publishers, 1992

[10] N. S. Jayant, P. Noll, Digital Coding of Waveforms, Prentice-Hall Inc., 1984 